\title{
Improved Diagnostic Accuracy Using Arterial Phase CT for Lateral Cervical Lymph Node Metastasis from Papillary Thyroid Cancer
}

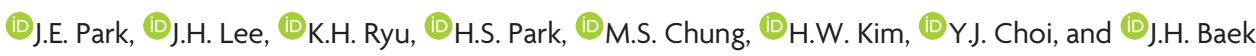

\begin{abstract}
BACKGROUND AND PURPOSE: Contrast-enhanced CT protocols for papillary thyroid cancer are yet to be optimized. Our aim was to compare the diagnostic accuracy of arterial phase CT and delayed-phase CT protocols for lateral cervical lymph node metastasis from papillary thyroid carcinoma by using the lymph node tissue attenuation.
\end{abstract}

MATERIALS AND METHODS: This retrospective study included 327 lateral cervical lymph nodes (177 metastatic and 150 benign) from 131 patients with papillary thyroid carcinoma (107 initially diagnosed and 24 recurrences). Patients underwent CT by using 1 of 3 protocols: a 70 -second (A) or a 35-second (B) delay with $100 \mathrm{~mL}$ of iodinated IV contrast or a 25 -second delay with $75 \mathrm{~mL}$ of IV contrast (C). Two readers independently measured and compared lymph node tissue attenuation between metastatic and benign lymph nodes. An area under the receiver operating characteristic curve analysis was performed to differentiate metastatic and benign lymph nodes after multiple comparison correction for clustered data and was compared across the protocols.

RESULTS: The difference in mean lymph node tissue attenuation between metastatic and benign lymph nodes was maximum in protocol $C(P<.001$ for both readers). Protocol $C$ showed the highest diagnostic performance (area under the receiver operating characteristic curve, 0.88-0.92) compared with protocol A (area under the receiver operating characteristic curve, $0.73-0.74, P<.001$ for both readers) and B (area under the receiver operating characteristic curve, .63-0.65, $P<.01$ for both readers). The sensitivity, specificity, positive predictive value, and negative predictive value of lymph node tissue attenuation by using a $99-\mathrm{HU}$ cutoff value were $83 \%-87 \%, 93.7 \%-$ $97.9 \%$, 95.1\%-97.3\%, and $81.2 \%-87 \%$.

CONCLUSIONS: A combination of 25-second delay CT and $75 \mathrm{~mL}$ of iodinated IV contrast can improve the diagnostic accuracy for lateral lymph node metastasis from papillary thyroid carcinoma compared with a combination of a 35- or 70-second delay with 100 -mL of iodinated IV contrast.

ABBREVIATIONS: $\mathrm{AUC}=$ area under the receiver operating characteristic curve; $\mathrm{CCA}=$ common carotid artery; $\mathrm{CTDI}_{\mathrm{vol}}=$ volume $\mathrm{CT}$ dose index; $\mathrm{DLP}=$ dose-length product; ICC = intraclass correlation coefficient; IJV = internal jugular vein; $L N=$ lymph node; LNTA = lymph node tissue attenuation; PTC = papillary thyroid carcinoma; US = ultrasound

D iagnosis of lateral cervical lymph node metastasis from papillary thyroid carcinoma (PTC) is clinically important in terms of preoperative surgical planning and predicting local tumor recurrence, particularly in high-risk patients. ${ }^{1-4}$ In preoperative planning, recent studies and guidelines have suggested that CT is complementary to ultrasound (US) in selected patients with

Received July 23, 2016; accepted after revision November 5.

From the Department of Radiology and Research Institute of Radiology, University of Ulsan College of Medicine, Asan Medical Center, Seoul, Korea.

Please address correspondence to Jeong Hyun Lee, MD, PhD, Department of Radiology and Research Institute of Radiology, University of Ulsan College of Medicine, Asan Medical Center, 86 Asanbyeongwon-Gil, Songpa-Gu, Seoul 138-736, Republic of Korea; e-mail: jeonghlee@amc.seoul.kr

三 Indicates article with supplemental on-line table

http://dx.doi.org/10.3174/ajnr.A5054 locally invasive primary tumor or clinically apparent metastatic lymph nodes. ${ }^{5-7}$ However, studies to test the diagnostic accuracy with CT have failed to prove its benefit over US for detecting lateral lymph node metastasis. ${ }^{8-11}$

Dynamic contrast-enhanced MR imaging and Doppler US studies have demonstrated increased tumor vascularity in metastatic lymph nodes (LNs) from PTC. ${ }^{12,13}$ The CT protocols among previous studies varied greatly from a scan delay of 35-60 seconds after contrast injection, ${ }^{8,9,14}$ and this delay might have resulted in heterogeneity of the diagnostic results. A CT protocol that best depicts increased tumor vascularity has been suggested in a study of parathyroid hormone-secreting lesions. ${ }^{15}$ An arterial phase protocol with a 25-second delay maximized the difference in tissue attenuation of parathyroid hormone-secreting lesions from the thyroid gland and benign LNs; tissue attenuation itself increased in venous phase CT. 


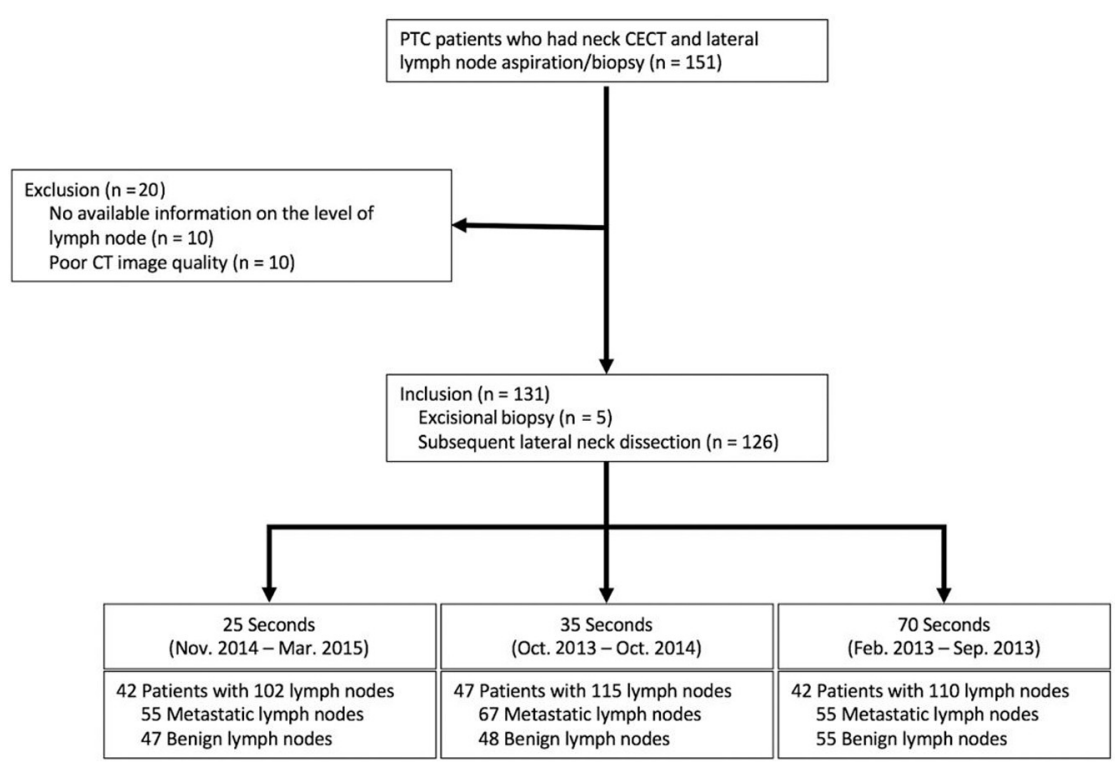

FIG 1. Flowchart of patient enrollment.

Because metastatic LNs from PTC have increased tumor vascularity, the use of the arterial phase CT might be better than venous phase CT in depicting lateral cervical LN metastasis from PTC.

In this study, we hypothesized that arterial phase CT would maximize the difference in tissue attenuation between metastatic and benign LNs in patients with PTC. We also hypothesized that quantification of LN tissue attenuation (LNTA) would be a useful tool in detecting lateral cervical LN metastasis from PTC. The purpose of this study was to compare the diagnostic accuracy between arterial phase CT and delayed-phase CT protocols for lateral cervical LN metastasis from PTC by using LNTA.

\section{MATERIALS AND METHODS Patient Selection}

This study was approved by our institutional review board. Data were collected retrospectively and were de-identified in compliance with the regulations of the Health Insurance Portability and Accountability Act. Informed consent was obtained from all patients before undergoing neck CT, US-guided biopsy, and/or an operation.

Among the patients who underwent CT between February 2013 and March 2015 found in the data base of our institution, 151 patients were identified as having primary or recurrent PTC with LN metastasis. Patients with recurrent PTC were those who had been treated with an operation alone and were later diagnosed as having a recurrence. The inclusion criteria were as follows: 1) patients with lateral cervical LN metastasis from PTC confirmed by US-guided aspiration/biopsy, 2) those who subsequently underwent selective neck dissection or excisional biopsy for metastatic lateral cervical LNs, and 3 ) those having available final histopathologic results. The exclusion criteria were as follows: 1) patients with histopathologic results that lacked information on the cervical level of the aspirated/biopsied LN (10 of 151 patients [6.6\%]), and 2) those with poor CT image quality because of motion or beam-hardening artifacts (10 of 151 patients [6.6\%]). Finally, 131 patients were included in this study.

\section{CT Protocols}

Imaging was performed by using a 128channel CT scanner (Somatom Definition Flash; Siemens, Erlangen, Germany) with tube voltages of 80 and $140 \mathrm{kVp}$. CT scanning began at the aorticopulmonary window and continued toward the skull base. CT was performed with the following parameters used consistently in all patients: $32 \times 0.6 \mathrm{~mm}$ detector collimation; 0.5 -second gantry rotation time; 1.0 pitch; 0.75 -mm-thick sections; 0.7-mm-thick section increments; and a $256 \times 256$ matrix. An automated dosereduction technique (CARE Dose4D; Siemens) was used. The volume CT dose index $\left(\mathrm{CTDI}_{\mathrm{vol}}\right)$ and dose-length product (DLP) were evaluated to assess radiation exposure.

Three different protocols for contrast-injection strategy and image-acquisition timing were used during CT. The protocols for patients with PTC at our institution were updated following a consensus among radiologists based on a literature review $^{15-17}$; the change was from a 70-second delay protocol to a 35- and 25-second scan delay. Protocol A consisted of a 70second scan delay after IV injection of 100-mL of iodinated contrast agent (February 2013 to September 2013; $n=42,35$ initially diagnosed and 7 with recurrent disease). Protocol B used a 35second scan delay after IV injection of $100 \mathrm{~mL}$ of iodinated contrast agent (October 2013 to October 2014; $n=47,40$ initially diagnosed and 7 with recurrent disease). Protocol $C$ consisted of a 25 -second scan delay after injection of $75 \mathrm{~mL}$ of iodinated contrast agent, followed by $50 \mathrm{~mL}$ of normal saline at the same rate to compensate for the small volume of contrast medium and to improve contrast medium use ${ }^{18}$ (November 2014 to March 2015; $n=42,32$ initially diagnosed and 10 with recurrent disease). For all scan delays, the same iodinated contrast agent, Ultravist (iopromide; Bayer HealthCare, Berlin, Germany), was injected at the same rate of $3.5 \mathrm{~mL} / \mathrm{s}$. The flowchart of patient enrollment is shown in Fig 1.

\section{Reference Standard and Histopathologic Assignment}

The final histopathologic reports of the US-guided aspiration/ biopsy or surgical neck dissection samples served as the reference standard for nodal metastasis. All cervical levels in a neck dissection specimen were labeled on the basis of the American Joint Committee on Cancer cervical regional lymph node level system $^{19}$; cervical LN levels were assigned as benign, metastatic, or mixed after correlation with the histopathologic reports. For example, if all the LNs in 1 level were histologically positive for metastasis, the cervical level was assigned as metastatic. If all the LNs in 1 level were histologically negative, the level was assigned as benign. If a level had both benign and metastatic LNs, the level was assigned as mixed. The results of the histopathologic assignment were subsequently used for LN matching and labeling on CT.

AJNR Am J Neuroradiol 38:782-88 Apr 2017 www.ajnr.org 783 


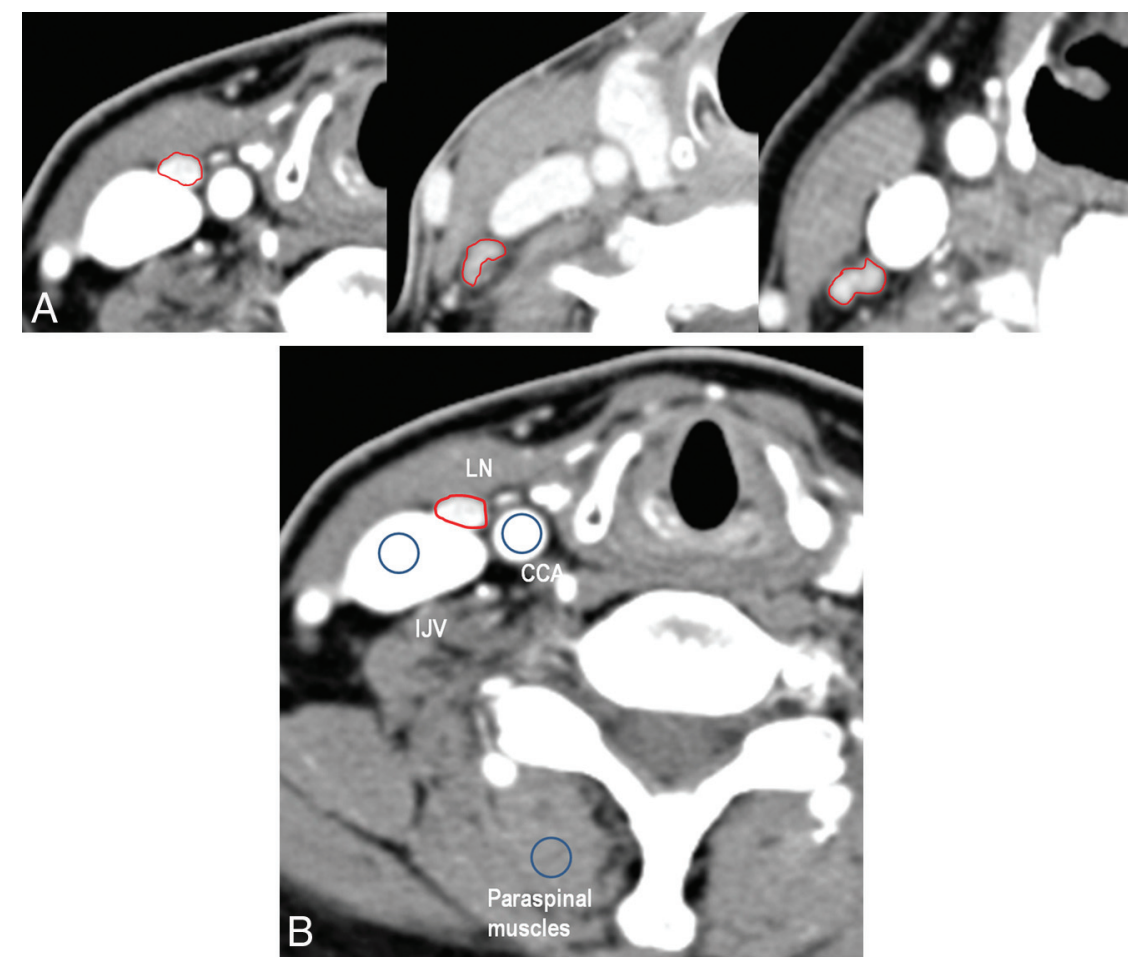

FIG 2. A, Contrast-enhanced CT scans obtained with protocol C, B, and A (from left to right) in different patients with papillary thyroid cancer. Note the enhancing metastatic lymph node in the right level III. B, A hyperenhancing lymph node in the right level III is shown in protocol C. The $\mathrm{ROI}\left(\mathrm{ROI}\right.$ area, $23.8 \mathrm{~mm}^{2}$; mean tissue attenuation, $172 \mathrm{HU}$ ) is drawn for a labeled metastatic lymph node. For normalization, a $60-\mathrm{mm}^{2}$ circular ROI was drawn on the ipsilateral CCA (mean CT value, $389 \mathrm{HU}$ ), IJV (mean CT value, $370 \mathrm{HU}$ ), and paraspinal muscle (mean CT value, $71.5 \mathrm{HU}$ ) on the same image. Lymph node tissue attenuation normalized to CCA, IJV, and paraspinal muscle was 0.44 , 0.46 , and 2.4 , respectively.

\section{LN Matching and Labeling on CT}

Lymph node matching and labeling on CT were performed on a PACS by a radiologist (J.H.L., with 14 years of experience in head and neck imaging), either by site-specific matching or surgicallevel matching. A LN confirmed by US-guided aspiration/biopsy was chosen and labeled on CT images by matching the images and reports of CT, US, and the final pathologic examination ("sitespecific matching"). When there was no site-specific information, LNs measuring $>5 \mathrm{~mm}$ in the minimum axis diameter and those assigned as benign or metastatic according to the previous histopathologic assignment were chosen and labeled on the CT images ("surgical-level matching"). All cervical-level LNs assigned as mixed were excluded from the final analysis.

\section{Measurement of LN Tissue Attenuation}

CT images with labeled LNs were transferred to ImageJ software (National Institutes of Health, Bethesda, Maryland) for assessment of LNTA (Hounsfield unit). Two readers (J.E.P. and K.H.R., with 2 years and 1 year of experience in head and neck imaging, respectively) were blinded to the clinicopathologic results and independently measured the LNTA of the labeled LNs and adjacent anatomic structures, including the common carotid artery (CCA), internal jugular vein (IJV), and paraspinal muscles. The ROI for the labeled LN was drawn to encompass the entire cortex, except for cystic change, necrosis, hilar fat/vessels, and calcification. A $60-\mathrm{mm}^{2}$ circular ROI was also drawn on the ipsilateral CCA, IJV, and paraspinal muscles at the same level as the labeled
LN (Fig 2). In addition, normalized LNTAs were calculated as the Hounsfield unit value of the entire LN divided by the Hounsfield unit value of the CCA, IJV, or paraspinal muscles.

Qualitative CT features (ie, calcification, cystic or necrotic change, and extranodal extension ${ }^{9,14}$ ) of the labeled LNs were assessed by an independent radiologist (Y.J.C., with 8 years of experience in head and neck imaging) who was blinded to the clinicopathologic results. An LN was categorized as positive when at least 1 of the qualitative CT features was present. We did not include the size criterion because LN size alone is an inaccurate criterion in young patients, who often have hyperplastic LNs, particularly at cervical levels I and II. ${ }^{20}$

\section{Statistical Analysis}

One-way analysis of variance or the Kruskal-Wallis test was performed to compare the characteristics of patients, LNs, and radiation exposure $\left(\mathrm{CTDI}_{\mathrm{vol}}\right.$ and DLP) among the 3 protocols. All parameters were initially assessed for normality by using the Kolmogorov-Smirnov test. The Student $t$ test was used to compare the parameters between benign and metastatic LNs.

Interreader agreement was assessed by using the intraclass correlation coefficient (ICC) with $95 \%$ confidence intervals and by applying a 2-way ICC with a random rater's assumption. On the basis of common criteria, measurement reliability was classified as excellent (ICC > 0.75), fair-to-good (ICC $=0.40-0.75)$, and poor $(\mathrm{ICC} \leq 0.40)$. $^{21}$

A receiver operating characteristic curve analysis was performed for each parameter to calculate the area under the receiver operating characteristic curve (AUC) and to determine the optimal cutoff for differentiating benign and metastatic LNs by each reader. To eliminate possible correlations from multiple measurements in the same patient, we used a logistic regression model with a generalized estimating equation model. ${ }^{22}$ We estimated the predicted probabilities of metastatic or benign LNs, and the estimated probability was then used as a marker for constructing the receiver operating characteristic curve and computing the area under the curve. We determined the cutoff values that maximized the sum of the sensitivity plus specificity as the points in the upper left-hand area for the receiver operating characteristic curve analysis for each parameter. For comparison of AUCs among protocols in different patient populations, a 2 -sample $Z$-test for comparing 2 means was used. For cross-validation of each AUC, we used leave-one-out cross-validation with bootstrap resampling on $\mathrm{R}$ statistical and computing software (http://www.r-project.org/) and analyzed the results by using the package pROC in $\mathrm{R}$. 
To assess the diagnostic performance of qualitative LN CT findings, we calculated the sensitivity, specificity, positive predictive value, negative predictive value, and accuracy by level-bylevel analysis.

Statistical analyses were performed by using statistical software (MedCalc, Version 10.2.0.0; MedCalc Software, Mariakerke, Belgium). All tests were 2 -sided. A $P$ value $<.05$ was considered significant.

\section{RESULTS}

\section{Patients}

The characteristics of the study patients are summarized in Table 1. Of 131 patients, 107 were initially diagnosed with PTC and 24 with recurrence. There were no significant differences among the 3 protocols in terms of age, sex, patient status, and median serum thyroglobulin level at baseline. Of the total 786 cervical levels ( $131 \times 6$ bilateral lateral neck levels), 110 mixed LN levels were excluded from the analysis.

The study population comprised a total of $327 \mathrm{LNs}$, including 177 metastatic LNs (149 initially diagnosed and 28 recurrent PTCs) and 150 benign LNs (135 initially diagnosed and 15 recurrent PTCs). Site-specific matching was found for 132 metastatic LNs and 22 benign LNs. Surgical-level matching was found for 45 metastatic LNs and 128 benign LNs. Of 177 metastatic LNs, 55 lymph nodes were evaluated by using protocol A; 67 lymph nodes, with protocol B; and 55 lymph nodes, with protocol C.

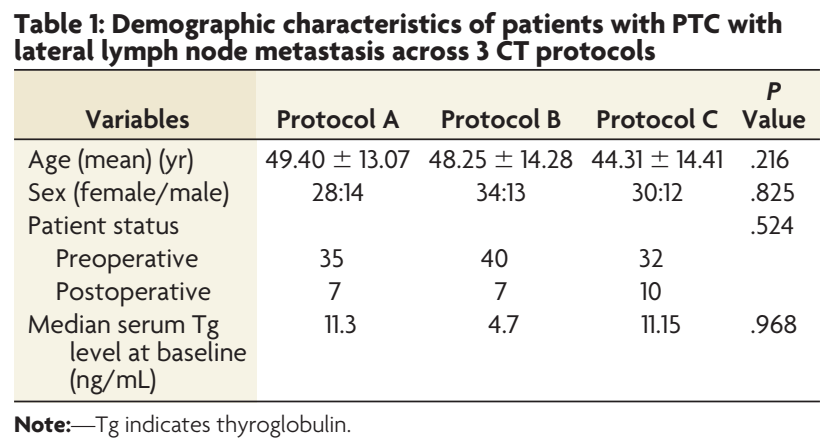

\section{Differences in LN Tissue Attenuations between Benign and Metastatic Lymph Nodes}

There was no significant difference in LNTA among the different LN levels. LNTA showed a significant difference between benign and metastatic LNs in all 3 protocols (all protocols, $P<.0001$ for reader 1; protocol B and $\mathrm{C}, P<.0001$; and protocol A, $P=.03$ for reader 2) (Table 2). LNTAs normalized to the CCA, IJV, and paraspinal muscle also showed a significant difference between benign and metastatic LNs in all 3 protocols (all protocols, $P<$ .001 for reader 1 ; protocol $\mathrm{B}$ and $\mathrm{C}, P<.001$; and protocol A, $P<$ .02 for reader 2 ).

When the differences in LNTA between benign and metastatic LNs were compared among the 3 protocols, protocol $\mathrm{C}$ showed the significantly largest LNTA of metastatic LNs as well as the significantly largest difference of LNTA between metastatic and benign LNs, followed by protocols B and A ( $P<.0001$ for both readers, Fig 3$)$.

The overall agreement in LNTA measurements in 2 readers was excellent ( ICC $=0.81$ ). The ICC was 0.76 for metastatic LNs and 0.60 for benign LNs.

\section{Comparison of the Diagnostic Performance among the 3 Protocols Using LN Tissue Attenuation}

In all tissue attenuation parameters assessed by both readers, protocol C showed significantly higher diagnostic performance compared with protocols B and A. With the LNTA, protocol C had the highest AUC $(0.8-0.92$; 95\% CI, 0.810.96 ) compared with protocol B (AUC, $0.73-0.74 ; P=.001$ for reader 1 and $P=.012$ for reader 2 ) and protocol A (AUC, $0.63-0.65 ; P<.001$ for both readers) (On-line Table and Fig 4). In protocol C, the optimal LNTA thresholds in differentiating metastatic from benign LNs were $96 \mathrm{HU}$ for reader 1 and $99 \mathrm{HU}$ for reader 2, respectively. With the $99-\mathrm{HU}$ cutoff from the receiver operating characteristic analysis, the sensitivity, specificity, positive predictive value, and negative predictive value for detecting metastatic LNs were $87.0 \%$ (95\% CI, 75.1\%-94.6\%), 97.9\% (95\% CI, 88.9\%-99.9\%), 97.3\% (95\% CI, $88.0 \%-99.9 \%$ ), and $87.0 \%$ (95\% CI, $75.1 \%-94.6 \%)$ for reader 1 ; the values for reader 2 were $83.0 \%$ (95\% CI, $70.2 \%-$ $91.9 \%), 93.7 \%$ (95\% CI, 82.8\%-98.7\%), 95.1\% (95\% CI, $82.8 \%-99.9 \%$ ), and $81.2 \%$ (95\% CI, 67.8\%-93.1\%), respectively. With a leave-one-out cross-validation, the LNTA

Table 2: Parameters of tissue attenuation between benign and metastatic lymph nodes among 3 protocols ${ }^{\mathrm{a}}$

\begin{tabular}{|c|c|c|c|c|c|c|c|c|c|}
\hline \multirow[b]{2}{*}{ Variables } & \multicolumn{3}{|c|}{ Protocol A } & \multicolumn{3}{|c|}{ Protocol B } & \multicolumn{3}{|c|}{ Protocol C } \\
\hline & $\begin{array}{l}\text { Metastatic } \\
(n=55)\end{array}$ & $\begin{array}{l}\text { Benign } \\
(n=55)\end{array}$ & $\begin{array}{c}P \\
\text { Value }\end{array}$ & $\begin{array}{c}\text { Metastatic } \\
(n=67)\end{array}$ & $\begin{array}{l}\text { Benign } \\
(n=48)\end{array}$ & $\begin{array}{c}P \\
\text { Value }\end{array}$ & $\begin{array}{c}\text { Metastatic } \\
(n=55)\end{array}$ & $\begin{array}{l}\text { Benign } \\
(n=47)\end{array}$ & $\begin{array}{c}P \\
\text { Value }\end{array}$ \\
\hline \multicolumn{10}{|l|}{ LNTA } \\
\hline Reader 1 & $107.2 \pm 28.6$ & $87.1 \pm 25.7$ & $<.0001$ & $109.1 \pm 40.2$ & $79.68 \pm 39.8$ & $<.0001$ & $136.3 \pm 41.6$ & $72.49 \pm 44.5$ & $<.0001$ \\
\hline Reader 2 & $109.8 \pm 27.5$ & $94.0 \pm 27.4$ & .03 & $123.7 \pm 39.8$ & $84.72 \pm 37.9$ & $<.0001$ & $133.2 \pm 43.8$ & $83 \pm 38.0$ & $<.0001$ \\
\hline \multicolumn{10}{|c|}{ Normalized LNTA to CCA } \\
\hline Reader 1 & $0.63 \pm 0.23$ & $0.49 \pm 0.13$ & $<.0001$ & $0.33 \pm 0.14$ & $0.25 \pm 0.08$ & $<.0001$ & $0.47 \pm 0.16$ & $0.26 \pm 0.05$ & $<.0001$ \\
\hline Reader 2 & $0.64 \pm 0.20$ & $0.52 \pm 0.12$ & .008 & $0.35 \pm 0.12$ & $0.28 \pm 0.12$ & $<.0001$ & $0.47 \pm 0.15$ & $0.29 \pm 0.14$ & $<.0001$ \\
\hline \multicolumn{10}{|c|}{ Normalized LNTA to IJV } \\
\hline Reader 1 & $0.58 \pm 0.15$ & $0.46 \pm 0.13$ & .0001 & $0.38 \pm 0.18$ & $0.28 \pm 0.09$ & $<.0001$ & $0.59 \pm 0.26$ & $0.33 \pm 0.17$ & $<.0001$ \\
\hline Reader 2 & $0.60 \pm 0.20$ & $0.55 \pm 0.12$ & .009 & $0.43 \pm 0.16$ & $0.30 \pm 0.15$ & $<.0001$ & $0.60 \pm 0.25$ & $0.37 \pm 0.24$ & $<.0001$ \\
\hline \multicolumn{10}{|c|}{$\begin{array}{l}\text { Normalized LNTA to } \\
\text { paraspinal muscle }\end{array}$} \\
\hline Reader 1 & $1.51 \pm 0.41$ & $1.20 \pm 0.34$ & $<.0001$ & $1.76 \pm 0.84$ & $1.26 \pm 0.31$ & $<.0001$ & $2.04 \pm 0.92$ & $1.13 \pm 0.25$ & $<.0001$ \\
\hline Reader 2 & $1.51 \pm 0.41$ & $1.36 \pm 0.29$ & .019 & $2.03 \pm 0.66$ & $1.40 \pm 0.36$ & $<.0001$ & $2.05 \pm 0.72$ & $1.28 \pm 0.49$ & $<.0001$ \\
\hline
\end{tabular}

${ }^{a}$ LNTAs were expressed as means. 


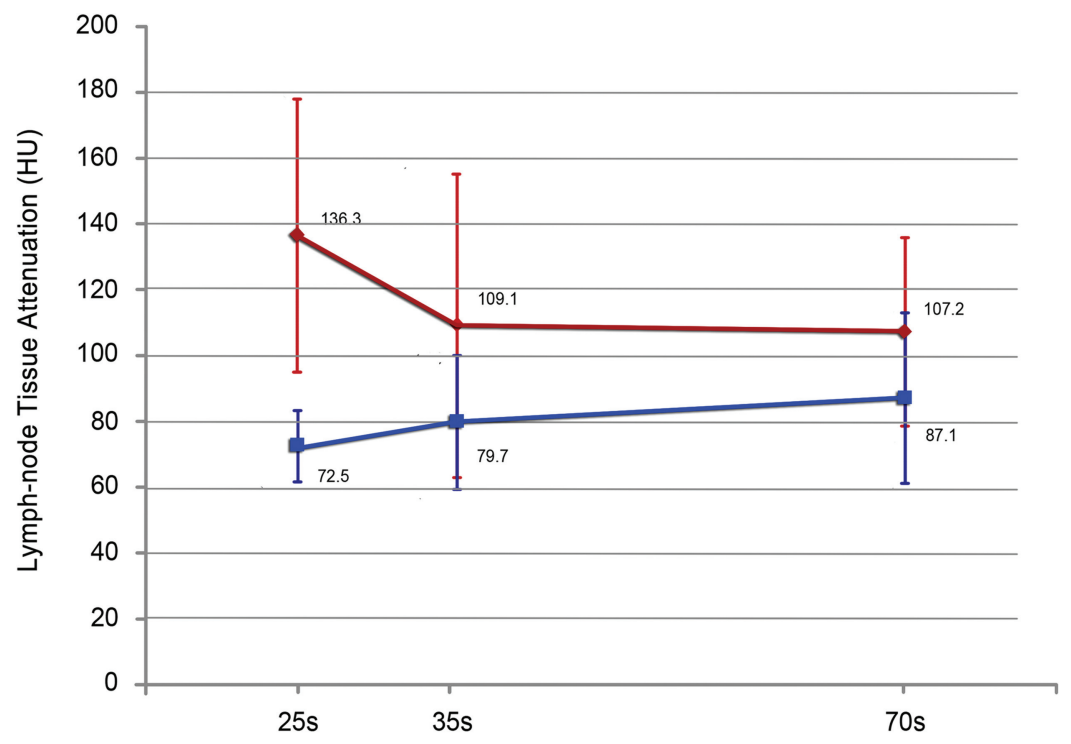

FIG 3. Comparison of the mean lymph node tissue attenuations assessed by reader 1 in protocols $\mathrm{A}, \mathrm{B}$, and $\mathrm{C}$. The red line represents lymph node tissue attenuations of metastatic lymph nodes, and the blue line represents those of benign lymph nodes. The largest tissue attenuation of metastatic lymph nodes and the difference in tissue attenuation between metastatic and benign lymph nodes are seen with protocol C, which has a 25-second scan delay, followed by protocol $\mathrm{B}$ (35-second delay) and protocol A (70-second delay) $(P<.0001)$.

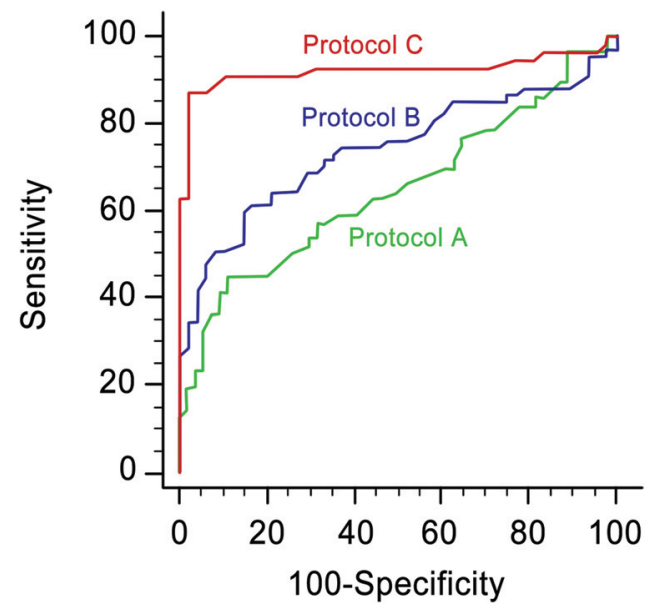

FIG 4. Graphs show receiver operating characteristic curves of the scan delay with protocols C ( 25 seconds, red line), B ( 35 seconds, blue line), and A (70 seconds, green line) assessed by reader 1, for differentiating metastatic and benign lymph nodes in patients with PTC.

Table 3: Characteristics and qualitative $\mathrm{CT}$ features of metastatic lymph nodes

\begin{tabular}{lccc}
\hline \multicolumn{1}{c}{$\begin{array}{c}\text { Variables } \\
\text { (No. of Lymph Nodes) }\end{array}$} & $\begin{array}{c}\text { Protocol A } \\
(\boldsymbol{n}=\mathbf{5 5})\end{array}$ & $\begin{array}{c}\text { Protocol B } \\
(\boldsymbol{n}=\mathbf{6 7})\end{array}$ & $\begin{array}{c}\text { Protocol C } \\
(\boldsymbol{n}=\mathbf{5 5})\end{array}$ \\
\hline Mean LN size $(\mathrm{mm})^{\mathrm{a}}$ & $9.0 \pm 3.09$ & $9.85 \pm 7.36$ & $10.35 \pm 5.27$ \\
Negative findings on CT & $31(56.4 \%)$ & $29(43.3 \%)$ & $23(41.8 \%)$ \\
$\begin{array}{l}\text { Positive findings on CT } \\
\text { Calcification }\end{array}$ & $8(14.5 \%)$ & $2(2.9 \%)$ & $11(20 \%)$ \\
Cystic/necrotic change & $15(27.3 \%)$ & $15(22.4 \%)$ & $13(23.6 \%)$ \\
$\quad$ Extranodal extension & $7(12.7 \%)$ & $10(14.9 \%)$ & $10(18.2 \%)$ \\
Level & & & \\
II & 6 & 9 & 5 \\
III & 18 & 18 & 21 \\
IV & 31 & 39 & 29 \\
\hline
\end{tabular}

${ }^{\mathrm{a}}$ Size of the lymph nodes were measured in the minimum axis diameter.

$\mathbf{7 8 6}$ Park Apr 2017 www.ajnr.org showed the same trend of highest AUC in protocol $\mathrm{C}$ in both readers.

Normalized LNTAs to the CCA and paraspinal muscles showed the same trend of diagnostic performance and showed the highest AUC in protocol C, compared with protocol B (normalized to the CCA, $P<.001$; and to the paraspinal muscles, $P=.001$ for reader 1 ; and to the CCA, $P=.003$; and to the paraspinal muscles, $P=.02$ for reader 2) and protocol A (normalized to the CCA, $P<.001$; and to the paraspinal muscles, $P<.001$ for reader 1 ; and to the CCA, $P<.001$; and to the paraspinal muscles, $P<$ .001 , for reader 2). Normalized LNTAs to the IJV showed higher AUC in protocol $\mathrm{C}$, compared with protocol $\mathrm{A}$ in both readers $(P=.012$ and $P=.008$, respectively) and in protocol $B$ in reader $1(P=.002)$, but were not significant with protocol $\mathrm{B}$ in reader 2 $(P=.065)$. In protocol $\mathrm{C}$, the diagnostic performance of the LNTA, when normalized to CCA, IJV, or the paraspinal muscles, did not differ significantly from that of the LNTA in both readers. All normalized parameters showed the highest diagnostic performance in protocol C.

\section{Diagnostic Performance of Qualitative CT Imaging Features for Metastatic Lymph Nodes}

Table 3 summarizes the characteristics and qualitative CT features of metastatic lymph nodes on the scanning protocols. There was no statistically significant difference among the protocols with regard to mean size and location of the LNs. Qualitative CT findings were absent in $46.8 \%$ ( 83 of 177) of the metastatic LNs. If we used the qualitative CT features in all 327 LNs, the overall diagnostic accuracy, sensitivity, specificity, positive predictive value, and negative predictive value for detecting metastatic LNs were 67.0\% (95\% CI, 62.0\%-71.0\%), 50.3\% (95\% CI, 45.7\%-54.0\%), $86.7 \%$ (95\% CI, 81.3\%-87.7\%), 81.7\% (95\% CI, 74.2\%-87.7\%), and $59.6 \%$ (95\% CI, 55.9\%-62.6\%), respectively.

\section{Radiation Exposure}

Both the mean $\mathrm{CTDI}_{\mathrm{vol}}$ and DLP were lowest in protocol C $\left(\mathrm{CTDI}_{\mathrm{vol}}, 10.8 \mathrm{mGy}\right.$; DLP, $\left.313.8 \mathrm{mGy} \times \mathrm{cm}\right)$ compared with protocol A $\left(\mathrm{CTDI}_{\mathrm{vol}}, 15.1 \mathrm{mGy}\right.$; DLP, $\left.458.8 \mathrm{mGy} \times \mathrm{cm}\right)$ and protocol B $\left(\right.$ CTDI $_{\text {vol }}, 11.8$ mGy; DLP, 329.9 mGy $\times$ cm) (Table 4). Compared with protocol A, both protocols $\mathrm{B}$ and $\mathrm{C}$ showed significantly lower radiation exposure based on both the CTDI ${ }_{\mathrm{vol}}$ and DLP (protocol B versus A, $P<.0001$; protocol C versus A, $P<$ $.0001)$. No statistically significant difference was found in the radiation exposure between protocols B and C.

\section{DISCUSSION}

Our study demonstrated that LNTA on protocol C, which comprised a combination of 25-second delay CT and $75 \mathrm{~mL}$ of iodin- 
Table 4: Mean radiation dose across the 3 protocols

\begin{tabular}{lcccc}
\hline \multicolumn{1}{c}{ Variables } & Protocol A & Protocol B & Protocol C & $\boldsymbol{P}$ Value \\
\hline $\mathrm{CTDI}_{\text {vol }}(\mathrm{mGy})$ & $15.1 \pm 2.2$ & $11.8 \pm 2.6$ & $10.8 \pm 2.1$ & $<.0001$ \\
$\mathrm{DLP}(\mathrm{mGy} \times \mathrm{cm})$ & $458.8 \pm 100.5$ & $329.9 \pm 83.4$ & $313.8 \pm 58.4$ & $<.0001$ \\
\hline
\end{tabular}

ated IV contrast, showed the best diagnostic performance among the other protocols for the assessment of lateral LN metastasis in patients with PTC. The differences between metastatic and benign LNs in both the LNTA and the normalized LNTAs were maximized with protocol C. The quantitative CT parameters with LNTA showed significantly better sensitivity, specificity, positive predictive value, and negative predictive value than the qualitative CT features. Our results suggest that measurement of LNTA with a 25-second scan delay can improve the diagnostic accuracy for lateral cervical LN metastasis in patients with PTC. The protocol is also potentially helpful in clinical practice, given its easy applicability and lower radiation exposure than protocol A.

Previous studies on the diagnostic performance of CT for PTC nodal disease showed a wide variation in sensitivity $(63.5 \%-79 \%)$ and specificity $(83 \%-95 \%) .{ }^{8,14,20}$ The discrepancy in these studies may have been caused by the subjective interpretation of strong cortical enhancement of metastatic LNs as well as the different CT examination protocols. We demonstrated that image acquisition and the strategy of contrast injection can affect the diagnostic performance of using LNTA on CT for detecting metastatic LNs from PTC. Our findings were also consistent with those of the recent work by Liu et $\mathrm{al}^{11}$ on dual-energy CT, which showed that quantitative measurement increased the diagnostic performance in detecting PTC nodal disease. However, our study had the advantage of easy applicability in daily clinical practice without the need for postprocessing or additional software.

The maximum difference in LNTA on arterial phase CT between metastatic and benign LNs is associated with increased tumor perfusion related to tumor angiogenesis and recruitment of capsular vessels in metastatic LNs. ${ }^{23-25}$ Previous dynamic contrast-enhanced MR imaging showed the value of quantitative analysis of tumor perfusion, which correlates with tumor response in patients with metastatic thyroid cancer. ${ }^{13}$ In our study, only $46.8 \%$ of metastatic LNs showed qualitative CT features suggestive of metastasis; these features included calcification, cystic or necrotic change, or extranodal extension. Relatively low sensitivities by using qualitative features for the detection of cervical lymph node metastasis can also be found on previous MR imaging or ${ }^{18}$ FDG-positron-emission tomography studies, which ranged from $30 \%$ to $40 \% .^{26}$ In patients with PTC, the use of quantitative LNTA parameters on arterial phase CT may be particularly beneficial for detecting LNs that lack qualitative CT findings.

The distribution of LNTA, which was presented as an SD, was greater in protocols $\mathrm{C}$ and $\mathrm{B}$ than in protocol $\mathrm{A}$ in both metastatic and benign LNs. The SD decreased after normalization by CCA and IJV, but not by the paraspinal muscles. A possible reason was that LNTA measurement can be greatly affected by heterogeneous patient hemodynamics, including cardiac output and local blood supply. ${ }^{18}$ LNTA normalized to CCA or IJV might be useful given that interindividual variation in perfusion factors might be decreased while maintaining high diagnostic performance.
The use of CT with iodinated contrast agents has been debated because it may decrease the effect of subsequent radioiodine therapy $^{27}$ and increase the radiation exposure. However, in recent guidelines, performing CT is supported because preoperative knowledge would significantly influence the surgical plan and outweighs a minor delay of up to 1 month in subsequent postoperative radioactive iodine ablation. ${ }^{7,28}$ In our study, the use of up to $25 \%$ less contrast material in a 25 -second scan delay protocol could reduce the radiation exposure in terms of both the CTDI ${ }_{\mathrm{vol}}$ and DLP, compared with the conventional head and neck CT of protocol A (a 70-second scan delay after a 100-mL iodinated contrast injection). In addition, the strategy of saline flushing after contrast injection not only decreased the radiation exposure but also improved the bolus geometry because of the decreased intravascular contrast medium dispersion ${ }^{18}$ and decreased artifacts from stagnated contrast agent within the subclavian or innominate vein. ${ }^{14,18}$

This study has several limitations. First, in addition to the retrospective nature of the study, each protocol was administered during a different time period, when the results could be influenced by factors outside the changes in the CT protocol. However, the possible systematic bias might have been minimized because the CT protocol used, except contrast-injection strategies and acquisition time, has long been standardized and strictly controlled by dedicated radiologists. Second, the sensitivity of our results might have been overestimated because we selected patients with PTC with lateral LN metastasis. Nevertheless, we believe that our study results clearly showed that compared with qualitative CT features, the quantitative parameter of LNTA might improve diagnostic accuracy and support visual analysis in the evaluation of lateral cervical LNs in patients with PTC. A third limitation was the inclusion of a relatively small number of benign LNs in level II, which frequently contains reactive lymph nodes from sinonasal or pharyngeal infection. Further studies might be necessary to compare differences in LNTAs between reactive and normal LNs. Finally, we did not study the same patients in all protocols; instead, there were 3 different patient populations. Performing all CT protocols in the same patient is unethical because of the excessive radiation exposure. Nevertheless, the patients in our study did not show any significant differences in clinical features across protocols. Future large-scale studies with random patient assignment to different protocols would further strengthen the findings of our study.

\section{CONCLUSIONS}

With a maximum difference in tissue attenuation between metastatic and benign LNs, the use of a combination of a 25 -second scan delay with 75-mL iodinated contrast injection can improve the diagnostic performance of CT for detecting lateral lymph node metastasis in patients with PTC. The use of arterial phase CT may be helpful in improving the detection of lateral cervical LN metastasis from PTC by providing higher sensitivity and specificity, as well as potentially lower radiation exposure compared with a CT protocol of a 70-second scan delay with a 100-mL iodinated contrast injection.

AJNR Am J Neuroradiol 38:782-88 Apr 2017 www.ajnr.org 


\section{REFERENCES}

1. Wu HW, Liu YH. 2012 NCCN guideline interpretation of the differentiated thyroid carcinoma [in Chinese]. Zhonghua Wai Ke Za Zhi 2012;50:675-77 CrossRef Medline

2. Gemsenjäger E, Perren A, Seifert B, et al. Lymph node surgery in papillary thyroid carcinoma. J Am Coll Surg 2003;197:182-90 CrossRef Medline

3. Ito Y, Tomoda C, Uruno T, et al. Preoperative ultrasonographic examination for lymph node metastasis: usefulness when designing lymph node dissection for papillary microcarcinoma of the thyroid. World J Surg 2004;28:498-501 CrossRef Medline

4. Kouvaraki MA, Lee JE, Shapiro SE, et al. Preventable reoperations for persistent and recurrent papillary thyroid carcinoma. Surgery 2004;136:1183-91 CrossRef Medline

5. Cooper DS, Doherty GM, Haugen BR, et al; American Thyroid Association (ATA) Guidelines Taskforce on Thyroid Nodules and Differentiated Thyroid Cancer. Revised American Thyroid Association management guidelines for patients with thyroid nodules and differentiated thyroid cancer. Thyroid 2009;19:1167-214 CrossRef Medline

6. Stack BC Jr, Ferris RL, Goldenberg D, et al; American Thyroid Association Surgical Affairs Committee. American Thyroid Association Consensus review and statement regarding the anatomy, terminology, and rationale for lateral neck dissection in differentiated thyroid cancer. Thyroid 2012;22:501-08 CrossRef Medline

7. Yeh MW, Bauer AJ, Bernet VA, et al; American Thyroid Association Surgical Affairs Committee Writing Task Force. American Thyroid Association statement on preoperative imaging for thyroid cancer surgery. Thyroid 2015;25:3-14 CrossRef Medline

8. Ahn JE, Lee JH, Yi JS, et al. Diagnostic accuracy of CT and ultrasonography for evaluating metastatic cervical lymph nodes in patients with thyroid cancer. World J Surg 2008;32:1552-58 CrossRef Medline

9. Choi JS, Kim J, Kwak JY, et al. Preoperative staging of papillary thyroid carcinoma: comparison of ultrasound imaging and CT. $A J R$ Am J Roentgenol 2009;193:871-78 CrossRef Medline

10. Choi JW, Yoon YH, Yoon YH, et al. Characteristics of primary papillary thyroid carcinoma with false-negative findings on initial (18)F-FDG PET/CT. Ann Surg Oncol 2011;18:1306-11 CrossRef Medline

11. Liu X, Ouyang D, Li H, et al. Papillary thyroid cancer: dual-energy spectral CT quantitative parameters for preoperative diagnosis of metastasis to the cervical lymph nodes. Radiology 2015;275:167-76 CrossRef Medline

12. Ahuja AT, Ying M, Yuen HY, et al. Power Doppler sonography of metastatic nodes from papillary carcinoma of the thyroid. Clin Radiol 2001;56:284-88 CrossRef Medline

13. Kloos RT, Ringel MD, Knopp MV, et al. Phase II trial of sorafenib in metastatic thyroid cancer. J Clin Oncol 2009;27:1675-84 CrossRef Medline

14. Kim E, Park JS, Son KR, et al. Preoperative diagnosis of cervical metastatic lymph nodes in papillary thyroid carcinoma: compari- son of ultrasound, computed tomography, and combined ultrasound with computed tomography. Thyroid 2008;18:411-18 CrossRef Medline

15. Gafton AR, Glastonbury CM, Eastwood JD, et al. Parathyroid lesions: characterization with dual-phase arterial and venous enhanced CT of the neck. AJNR Am J Neuroradiol 2012;33:949-52 CrossRef Medline

16. Bahl M, Sepahdari AR, Sosa JA, et al. Parathyroid adenomas and hyperplasia on four-dimensional CT scans: three patterns of enhancement relative to the thyroid gland justify a three-phase protocol. Radiology 2015;277:454-62 CrossRef Medline

17. Raghavan P, Durst CR, Ornan DA, et al. Dynamic CT for parathyroid disease: are multiple phases necessary? AJNR Am J Neuroradiol 2014;35:1959-64 CrossRef Medline

18. Bae KT. Intravenous contrast medium administration and scan timing at CT: considerations and approaches. Radiology 2010;256: 32-61 CrossRef Medline

19. Greene FL. American Joint Committee on Cancer, American Cancer Society. AJCC Cancer Staging Handbook: From the AJCC Cancer Staging Manual. 6th ed. New York: Springer-Verlag; 2002

20. Lesnik D, Cunnane ME, Zurakowski D, et al. Papillary thyroid carcinoma nodal surgery directed by a preoperative radiographic map utilizing CT scan and ultrasound in all primary and reoperative patients. Head Neck 2014;36:191-202 CrossRef Medline

21. Büsing KA, Kilian AK, Schaible T, et al. Reliability and validity of MR image lung volume measurement in fetuses with congenital diaphragmatic hernia and in vitro lung models. Radiology 2008;246: 553-61 CrossRef Medline

22. Beam CA. Analysis of clustered data in receiver operating characteristic studies. Stat Methods Med Res 1998;7:324-36 CrossRef Medline

23. Na DG, Lim HK, Byun HS, et al. Differential diagnosis of cervical lymphadenopathy: usefulness of color Doppler sonography. AJR Am J Roentgenol 1997;168:1311-16 CrossRef Medline

24. Wu CH, Chang YL, Hsu WC, et al. Usefulness of Doppler spectral analysis and power Doppler sonography in the differentiation of cervical lymphadenopathies. AJR Am J Roentgenol 1998;171:503-09 CrossRef Medline

25. Ying M, Bhatia KS, Lee YP, et al. Review of ultrasonography of malignant neck nodes: grayscale, Doppler, contrast enhancement and elastography. Cancer Imaging 2014;13:658-69 CrossRef Medline

26. Jeong HS, Baek CH, Son YI, et al. Integrated 18F-FDG PET/CT for the initial evaluation of cervical node level of patients with papillary thyroid carcinoma: comparison with ultrasound and contrast-enhanced CT. Clin Endocrinol (Oxf) 2006;65:402-07 CrossRef Medline

27. Nygaard B, Nygaard T, Jensen LI, et al. Iohexol: effects on uptake of radioactive iodine in the thyroid and on thyroid function. Acad Radiol 1998;5:409-14 CrossRef Medline

28. Haugen BR, Alexander EK, Bible KC. 2015 American Thyroid Association management guidelines for adult patients with thyroid nodules and differentiated thyroid cancer. Thyroid 2016;26:1-133 CrossRef Medline 